\title{
A Large Gastroesophageal Junction Mass in an Elderly Caucasian Male
}

\author{
Nathaniel A Parker*, Hussein Agha Y and Jeremy M Duestsch \\ Department of Internal Medicine, University of Kansas School of Medicine, USA
}

*Corresponding author: Nathaniel A Parker, Department of Internal Medicine, University of Kansas School of Medicine, Wichita, KS, USA

Received Date: May 26, 2020

Published Date: June 11, 2020

\begin{abstract}
Abbreviations: CT: Computed Tomography; GEJ: Gastroesophageal Junction; GI: Gastrointestinal; IHC: Immunohistochemical; MART-1: Melanoma Antigen Recognized by T-cells; PSA: Prostate-specific Antigen; PET: Positron Emission Tomography; BRAF: B-Raf; NRAS: Neuroblastoma RAS; GNAQ: G Protein Subunit Alpha Q; GNA11: G Protein Subunit Alpha 11; c-KIT: Receptor Tyrosine Kinase.
\end{abstract}

\section{Case Description}

A 90-year-old Caucasian male presented to the emergency department with the chief complaint of solid-food dysphagia and unintentional weight loss. Symptom onset began three months prior to his initial presentation and had been progressively worsening. Vital signs and measurements, and physical examination were unremarkable. Serum laboratory evaluation was primarily nonrevealing, except for his hemoglobin which was approximately $8 \mathrm{gm} / \mathrm{dL}$ (reference range $12-16 \mathrm{gm} / \mathrm{dL}$ ) and positive stool guaiac testing. Chest and abdominopelvic CT scans with contrast were obtained for further elucidation of the patient's symptoms. Imaging showed a large, invasive esophageal mass. The mass originated at the level of the carina in the mid-esophagus and extended approximately $15 \mathrm{~cm}$ inferiorly into the gastric cardia, fundus, and body. At its maximum dimensions of 6 x $9.4 \mathrm{~cm}$ coronally, the mass was centered at the GEJ, and was associated with regional lymphadenopathy concerning for metastatic disease (Figure 1). Subsequently, he underwent an esophagogastroduodenoscopy which revealed a large, ulcerated mass at the GEJ resulting in near total esophageal occlusion. Core biopsies were collected during upper GI endoscopy to confirm the diagnosis. Histopathology revealed sheets of malignant cells. IHC staining was positive for MART-1. Pankeratin, CD56, synaptophysin, chromogranin, CDX2, p63, and PSA immunostaining was negative. Together with imaging evidence and pathology, primary melanoma of the GEJ was diagnosed. The patient was dismissed from the hospital and instructed to follow up with his local oncologist for a PET/CT scan and genetic testing. However, the patient expired before these subsequent tests could be performed. 

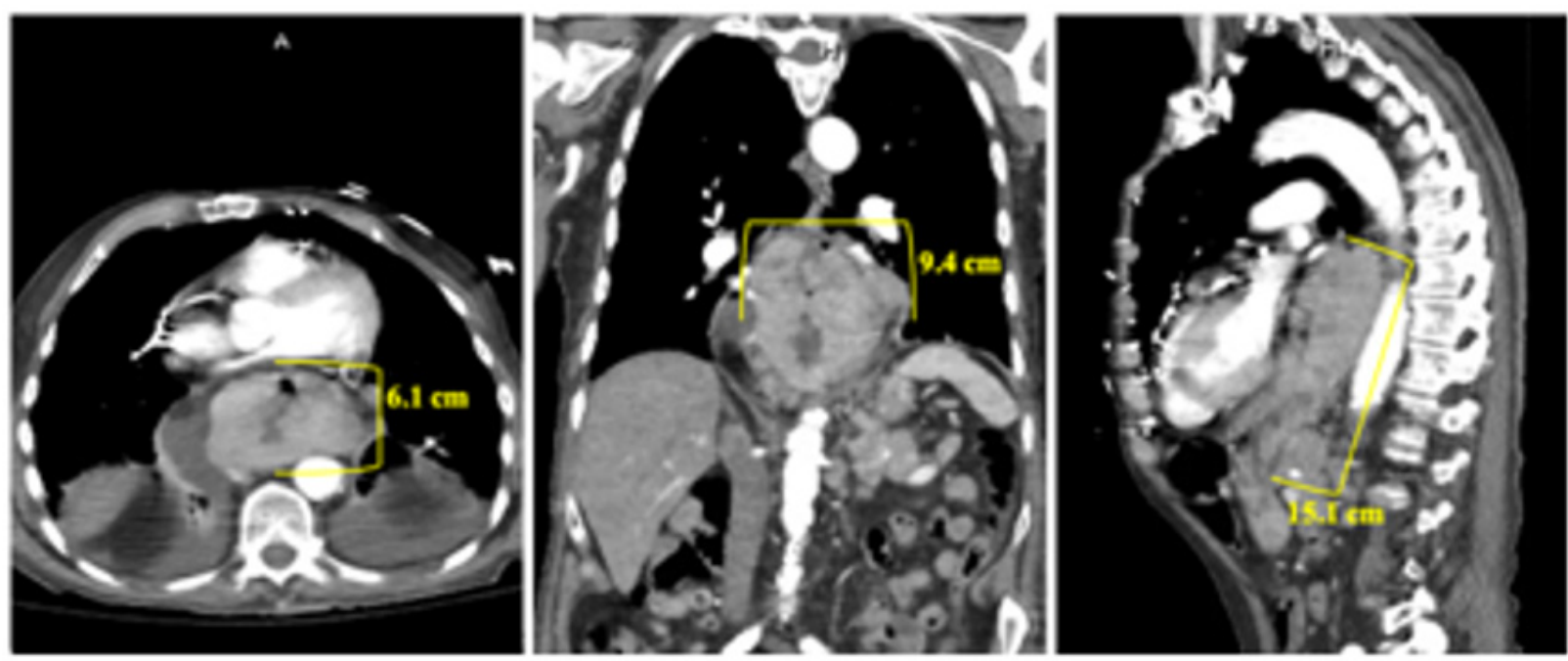

Figure 1: CT scans with contrast demonstrate a large mass centered around the GEJ.

\section{Discussion}

Primary mucosal melanoma is an exceedingly rare phenomenon. Although dispersed normally in low numbers, melanocytes are present in non-sun-exposed regions of the body, such as the oral and nasal cavity, paranasal sinuses, larynx, esophagus, anorectal canal, vagina, and cervix [1]. However, the etiopathogenesis of benign melanocytes transforming into melanoma remains poorly understood. Less than $3 \%$ of all melanomas are non-cutaneous and diagnosed with a unknown primary site [2]. Recently, morphologically identical but characteristically diverse melanoma variants have emerged. Mucosal melanoma, melanoma of unknown primary, and melanoma of soft parts represent three unique melanoma variants with distinct differences in typical location of origin, pathogenesis, progression, aggressiveness, genetic profile, response to therapy, and survival rates. Melanoma of unknown primary occurs more commonly in middle-aged males, and typically is diagnosed in lymph nodes and subcutaneous tissue (70 - 90\%) [2]. In contrast, the peak incidence of melanoma of soft parts occurs in the second through fourth decades of life. This variant preferentially involves the extremities, especially the legs, and is often found to be deeply infiltrative and adherent, such as being bound to surrounding tendons [3]. This case not only lacked the opportunity for PET scans to be used for staging and evaluation of distant metastasis, but also genomic profiling could not be performed. However, no universal staging system has been established for any of the non-cutaneous melanoma variants. Imaging supported extensive, local gastroesophageal tumor involvement. The patient denied previous skin excisions or current cutaneous lesions, and there was no radiologic evidence of lymphadenopathy in the sun-exposed lymph node groups (e.g. head and neck, axillary, and inguinal). Thus, although it is difficult to be entirely dogmatic on this point, the lesion most likely was a primary mucosal melanoma.
Primary mucosal melanomas are exceedingly rare, and account for approximately $1 \%$ of all melanomas [1]. Mucosal melanomas can arise from the gastrointestinal tract, but this is uncommon, whereas nearly $50 \%$ originate from anorectal tissue [1]. Gastroesophageal melanoma is an extraordinarily rare entity that represents less than $10 \%$ and $0.1 \%$ of primary mucosal and all melanomas, respectively [1]. They preferentially occur in females, which is thought to be due to a large majority of mucosal melanomas arising from the genital tract [4]. Although less pronounced compared to cutaneous melanoma, a Caucasian predominance exists [5]. Likely due to inordinately low incidence rates, no risk factors for development have been identified. Viral carcinogenesis studies have yet to find any associations [1]. However, chronic exposure to tobacco smoke and formaldehyde have been postulated to be a possible risk factor for oral and sinonasal mucosal melanoma, respectively [1].

Melanoma of unknown primary is predominantly hypothesized to develop from the spontaneous regression of melanoma from a known primary site [2]. Thus, with cutaneous melanoma accounting for more than $97 \%$ of all melanomas, melanoma of unknown primary and cutaneous melanoma typically share similar genetic profiles consisting of BRAF and NRAS mutations [2]. Either GNAQ or GNA11 gene mutations almost exclusively occur in uveal melanomas [6,7]. However, mucosal melanomas harbor a unique genomic profile characterized by alterations in the c-KIT gene [89]. Since the discovery of c-KIT aberrations in primary mucosal melanomas, KIT-inhibitors like imatinib and sunitinib have provided the opportunity for targeted therapy. However, not all mucosal melanoma tumors harbor c-KIT mutations [1]. Furthermore, due to this entity's exceedingly low incidence rates, large clinical trials studying the efficacy of targeted therapeutic agents are unlikely feasible. KIT-inhibitors have been shown to be effective in certain studies, but overall, the five-year survival for mucosal melanoma is significant less than cutaneous melanoma [10-12]. 


\section{Conclusion}

Written informed consent for publication of their clinical details and clinical images was obtained from the patient.

\section{Acknowledgement}

None.

\section{Conflict of Interest}

No conflict of interest.

\section{References}

1. Mihajlovic M, Vlajkovic S, Jovanovic P, Stefanovic V (2012) Primary mucosal melanomas: a comprehensive review. Int J Clin Exp Pathol 5(8): 739-753.

2. Scott JF, Gerstenblith MR (2018) Melanoma of Unknown Primary. In Scott JF, Gerstenblith MR (eds.), Noncutaneous Melanoma [Internet] Brisbane (AU): Codon Publications.

3. Couturier J (1999) Soft tissue tumors: Malignant melanoma of soft parts. Atlas Genet Cytogenet Oncol Haematol 3(4): 207- 208.

4. McLaughlin CC, Wu XC, Jemal A, Martin HJ, Roche LM, et al. (2005) Incidence of noncutaneous melanomas in the U.S. Cancer 103(5): 1000-1007.
5. $\mathrm{Hu}$ DN, Yu G, McCormick SA, Finger PT (2008) Population-based incidence of conjunctival melanoma in various races and ethnic groups and comparison with other melanomas. Am J Ophthalmol 145(3): 418-423.

6. Van Raamsdonk CD, Bezrookove V, Green G, Jürgen Bauer, Lona Gaugler, et al. (2009) Frequent somatic mutations of GNAQ in uveal melanoma and blue naevi. Nature. 2009;457(7229): 599-602.

7. Van Raamsdonk CD, Griewank KG, Crosby MB, Maria C Garrido, Swapna Vemula, et al. (2010) Mutations in GNA11 in uveal melanoma. N Engl J Med 363(23): 2191-2199.

8. Curtin JA, Busam K, Pinkel D, Bastian BC (2006) Somatic activation of KIT in distinct subtypes of melanoma. J Clin Oncol 24(26): 4340-4346.

9. Beadling C, Jacobson-Dunlop E, Hodi FS, Claudia Le, Andrea Warrick, et al. (2008) KIT gene mutations and copy number in melanoma subtypes. Clin Cancer Res 14(21): 6821-6828.

10. Hodi FS, Friedlander P, Corless CL, Michael C Heinrich, Suzanne Mac Rae, et al. (2008) Major response to imatinib mesylate in KIT-mutated melanoma. J Clin Oncol 26(12): 2046-2051.

11. Minor DR, Kashani-Sabet M, Garrido M, O’Day SJ, Hamid O, et al. (2012) Sunitinib therapy for melanoma patients with KIT mutations. Clin Cancer Res 18(5): 1457-1463.

12. Chang AE, Karnell LH, Menck HR (1998) The National Cancer Data Base report on cutaneous and noncutaneous melanoma: a summary of 84,836 cases from the past decade. Cancer 83(8): 1664-1678. 